\title{
PSICOLOGIA IBEROAMERICANA
}

\section{Psicología Iberoamericana}

ISSN: 1405-0943

psicología.iberoamericana@uia.mx

Universidad Iberoamericana, Ciudad de

México

México

Sánchez Estrada, Marcela; González Forteza, Catalina; Robles García, Rebeca; Andrade Palos,

Patricia

Desarrollo y evaluación psicométrica de un índice de espiritualidad para adultos mayores en México

Psicología Iberoamericana, vol. 20, núm. 2, julio-diciembre, 2012, pp. 41-48

Universidad Iberoamericana, Ciudad de México

Distrito Federal, México

Disponible en: http://www.redalyc.org/articulo.oa?id=133928816006

- Cómo citar el artículo

- Número completo

- Más información del artículo

- Página de la revista en redalyc.org

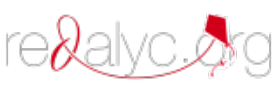

Sistema de Información Científica

Red de Revistas Científicas de América Latina, el Caribe, España y Portugal

Proyecto académico sin fines de lucro, desarrollado bajo la iniciativa de acceso abierto 


\title{
Desarrollo y evaluación psicométrica de un índice de espiritualidad para adultos mayores en México
}

\author{
Development and Psychometric Evaluation of a Spirituality \\ Index for Elders in Mexico
}

\author{
Marcela Sánchez Estrada* \\ instituto Nacional de Salud Pública de México \\ Catalina González Forteza \\ Rebeca Robles García \\ instituto nacional de Psiquiatría Ramón de la Fuente Muñiz \\ Patricia Andrade Palos \\ Universidad Nacional Autónoma de México
}

\section{RESUMEN}

Los objetivos del presente estudio fueron el desarrollo de un índice para evaluar la espiritualidad en Adultos Mayores (AM) en México, y la evaluación de sus características psicométricas. Los reactivos se basaron en experiencias cotidianas de AM. Participaron 355 AM que acudieron al Instituto Nacional de las Personas Adultas Mayores (Inapam), con $\mathrm{M}=68$ años y $\mathrm{DE}=7.9$. Se aplicaron: la versión piloto de Espiritualidad y las escalas Depresión geriátrica y Bienestar subjetivo. El análisis factorial exploratorio del instrumento final arrojó una estructura de un solo factor, con valor Eigen= 4.64, que explicó el 77\% de la varianza. El análisis factorial confirmatorio mostró un modelo adecuado a los datos $\left(\mathrm{X}^{2}=12.47, g l=9, p=1.88\right)$. El alfa de Cronbach fue .94; se obtuvo una correlación negativa $(r=-.20, p=.01)$ con Depresión, y positiva $(r=.11, p=.01)$ con Bienestar. Los resultados muestran un índice breve de espiritualidad válido y confiable útil para la población mexicana de AM.

Palabras clave: Adultos Mayores, Bienestar, Espiritualidad, Evaluación Psicométrica, México.

\section{ABSTRACT}

The aims of present study were to design an index of spirituality for elders in Mexico and evaluate its psychometric properties. The items were based on everyday experiences of elders. The participants were 355 elders that asked services at the National Institute of Older Persons (Inapam), with $M=68$ years old and $S D=7.9$. The application included: the pilot version of Spirituality, and the Geriatric depression, and Subjective well-being scales. The exploratory factor analysis of the final instrument showed one factor with an Eigen value $=4.64$ that explained $77 \%$ of the variance. Confirmatory factor analysis showed an adequate model fit to the data $(X 2=12.47, g l=9, p=1.88)$. The internal consistency was adequate Cronbach $=.94$. Spirituality index had negative correlation with Depression $(r=-.20, p=.01)$; and positive with Subjective well-being $(r=.11, p=.01)$. The index of Spirituality is a valid and reliable instrument adequate to Mexican elders.

Keywords: Elders, Mexico, Psychometric Evaluation, Spirituality, Well-being.

\footnotetext{
* Las autoras agradecen al Instituto Nacional de las Personas Adultas Mayores (Inapam) por todas las facilidades otorgadas para la aplicación de los instrumentos en sus instalaciones. A la Universidad Nacional Autónoma de México (UNAM) por la asesoría y colaboración académica, y al Consejo Nacional de Ciencia y Tecnología (Conacyt) por la beca 164664 para la realización de estudios doctorales otorgada a la primera autora de este trabajo. La correspondencia en relación con este artículo debe dirigirse a Marcela Sánchez Estrada,

Dirección: Instituto Nacional de Salud Pública. 7a. Cerrada de Fray Pedro de Gante, No. 50, Col. Sección XVI, Delegación Tlalpan, C.P. 14000, México D. F. Dirección electrónica: mar9977@yahoo.com
} 


\section{INTRODUCCIÓN}

El incremento del grupo de Adultos Mayores (AM) y las proyecciones demográficas para los siguientes años a nivel mundial han propiciado un enfoque hacia el fomento del bienestar sobre la visión tradicional de disminución de capacidades y funcionalidad que se espera se presenten al incrementarse la edad. La perspectiva tradicional de detrimento de las capacidades y desarrollo de patologías es un escenario complicado para el futuro; sin embargo, hay evidencia de que se pueden realizar acciones para mejorar el estado de salud y bienestar de los AM al considerar sus habilidades, condiciones reales y aspectos positivos (Fernández-Ballesteros, 2009). La Organización Mundial de la Salud (Organización de las Naciones Unidas, 2002) promueve mantener la capacidad funcional durante la vida por medio de la activación de los AM, conservando su independencia y previniendo la discapacidad tanto como sea posible (Cloos et al., 2001; Oxley, 2009).

Desde esta perspectiva, el bienestar está relacionado con aquellas características particulares de los AM que fomentan su actividad y funcionalidad, de acuerdo con su condición (Buendía \& Riquelme, 1997; Vázquez \& Hervás, 2009). Una de estas características es la espiritualidad, ya que se observa su incremento en los grupos de mayor edad, en comparación con grupos más jóvenes (Bailly \& Roussiau, 2010).

Este aumento se ha explicado por los cambios en los ámbitos social, económico y de salud particulares de la tercera edad, influenciado también por aspectos culturales (Rivera, 2007).

En la última década, en el estudio de la espiritualidad se ha enfatizado su diferenciación con respecto a la religiosidad, de la cual se había considerado sinónimo. Recientemente se han llevado a cabo estudios para identificar las similitudes y diferencias entre estos dos conceptos (Gorge, Larson, Koenig \& McCullough, 2000; Koenig, 2004); encontrando que cada uno tiene características específicas que los distinguen (Peterson \& Seligman, 2004).

Entre las diferencias, se ha identificado que la religiosidad se vincula con un sistema de creencias y acciones relacionados con cultos organizados socialmente, con instituciones formales y prácticas grupales dentro de éstas, entre las que destaca la aceptación y seguimiento de dichas creencias, prácticas y rituales tradicionales de la doctrina como parte de la pertenencia a la misma (Lowis, Eduards \& Burton, 2009; Rivera, 2007). Por otro lado, la espiritualidad se relaciona con el significado personal del sentido de la vida y de la relación con un ser supremo; y es el resultado de un estado interno, basado en las experiencias personales, asociado con la dimensión de conciencia y trascendencia de la vida y no necesariamente vinculado con lo social o con algún dogma en particular; de esta manera se puede identificar a quienes se consideran espirituales, pero no religiosos (Peterson \& Seligman, 2004; Sawatzky, Ratner \& Chiu, 2005).

La espiritualidad, en relación con el bienestar de los $\mathrm{AM}$, se ha vinculado con la reducción de índices de mortalidad, enfermedades cardiovasculares, hipertensión, depresión, ansiedad (Ardelt, 2003; Eun-Kyoung, 2007); así como con la mejora en el funcionamiento cognitivo y manejo del estrés (Bailly \& Roussiau, 2010); el control en enfermedades crónicas y agudas, y de estilos de vida saludables (Lee \& Newberg, 2005; Mueller, Plevak \& Rummans, 2001; Powell, Shahabi \& Thoresen, 2003). Estos efectos son variables en cuanto a la magnitud y dirección (Daaleman \& Frey, 2004; Sawatzky, et al., 2005; Smith, Poll \& McCullough, 2003), lo que puede deberse a la tendencia, aún presente, de considerar sinónimos a la espiritualidad y la religiosidad (Williams \& Sternthal, 2007), así como a la operacionalización de la espiritualidad y a los instrumentos empleados para evaluarla (Herbert, Weinstein, Martinre \& Schulz, 2006; Sawatzky, et al., 2005).

La diversidad de los instrumentos para evaluarla es amplia, desde preguntas únicas y específicas, así como de la percepción del nivel de espiritualidad (Powell et al., 2003; Sawatzky et al., 2005; Williams \& Sternthal, 2007), hasta instrumentos multidimensionales (EunKyoung, 2007).

La evaluación de la relación entre espiritualidad y bienestar no está consensada debido básicamente a los diferentes instrumentos que se han usado para evaluarla. Existen estudios que indican que la espiritualidad sí tiene un efecto positivo en el bienestar de las personas (Bailly \& Roussiau, 2010; Daaleman \& Frey, 2004; Sawatzky et al., 2005), mientras que otros afirman que no hay relación (Ardelt, 2003; Eun-Kyoung, 2007; Herbert et al., 2006). 
De la amplia gama de instrumentos disponibles, se encuentran, entre los más empleados con AM:

La Escala de Bienestar Espiritual de la Evaluación Funcional de Enfermedades Crónicas -FACIT-SP por sus siglas en inglés (Cella et al., 1993), que se desarrolló para evaluar el bienestar en pacientes con diversas enfermedades crónicas. Originalmente, el instrumento constaba de cuatro áreas: bienestar físico, social/familiar, emocional y funcional. Después se incluyó una subescala de espiritualidad con dos factores: uno relacionado con el sentido y significado, paz y propósito de la vida (con un alfa de Cronbach $=.81$ ) y el segundo (con un alfa de Cronbach $=.88$ ) derivado de la fe personal (Brady, Peterman, Fitchett \& Cella, 1999).

La Escala Multidimensional de Religiosidad y Espiritualidad (MMRS por sus siglas en inglés) con seis subescalas (Fetzer Institute, 1999), para evaluar la percepción de Dios o de lo divino en la vida cotidiana (alfa de Cronbach $=.91$ ), valores religiosos (alfa de Cronbach $=.64$ ), perdón (alfa de Cronbach $=.66$ ), prácticas religiosas privadas (alfa de Cronbach $=.72$ ), habilidades de enfrentamiento religioso/espiritual (alfa de Cronbach $=.81$ ), y apoyo religioso (alfa de Cronbach=.86). La MMRS se ha aplicado a muestras de AM con índices de confiabilidad muy parecidos a los originales (Eun-Kyoung, 2007).

El Índice de Bienestar Espiritual (SIWB por sus siglas en inglés) está compuesto por dos factores: uno sobre autoeficacia, y el segundo referente al sentido de la vida (Daaleman, Frey, Wallace \& Studenski, 2002). El primero obtuvo un alfa de $\mathrm{Cronbach}=83$; y el segundo un alfa de Cronbach=80.

La Escala Diaria de Experiencias Espirituales (DSES por sus siglas en inglés), está integrada por un sólo factor que evalúa la percepción de trascendencia (presencia de Dios o divinidad) en la vida cotidiana (Underwood \& Teresi, 2002). Aunque en la escala se emplea el sustantivo Dios, los autores sugieren cambiarlo para no relacionar la espiritualidad con una doctrina en particular. La escala obtuvo una alfa de Cronbach $=.93$.

La escala de Bienestar Espiritual (SWB por sus siglas en inglés) está integrada por dos subescalas: bienestar religioso en torno a la relación con Dios (con un alfa de Cronbach=.96), ybienestar existencial (con un alfa de Cronbach=.86), que incluye la adaptación consi- go mismo, la comunidad y el ambiente (Ellison, 1983). La subescala de bienestar religioso fue validada en México (EBE por sus siglas en español); evalúa la satisfacción de la relación con Dios, y su contraparte: la insatisfacción en esta misma relación. En México la EBE obtuvo un alfa de Cronbach=.84 (Rivera-Ledesma \& Montero-López, 2007).

La Organización Mundial de la Salud (OMS) consideró la espiritualidad en el estudio de la calidad de vida en la escala desarrollada para evaluar la Calidad de Vida WHOQOL 100 (por sus siglas en inglés y el número de reactivos de la versión larga), donde incluyó cuatro reactivos sobre espiritualidad/religiosidad y creencias personales; obteniendo un alfa de Cronbach $=.80$ en México (González-Celis, Tron \& Chávez, 2009).

Dado que la gran mayoría de los instrumentos previamente descritos incluyen aspectos de espiritualidad y de religiosidad como parte del mismo constructo; o bien factores no directamente relacionados con la espiritualidad como la autoeficacia (Daaleman et al., 2002), se consideró necesario construir un instrumento que permitiera evaluar la espiritualidad distinguida de la religiosidad.

Cabe señalar que aunque hay instrumentos que contienen varios factores, la muldimensionalidad no implica necesariamente una profundización en el concepto de espiritualidad (Idler, 2003). Uno de los aspectos más importantes a considerar para conceptualizar la espiritualidad es su separación de la religiosidad; de tal manera que cualquier persona, independientemente de la afiliación religiosa que tenga y del cumplimiento de las actividades relacionadas con dicha religión, pueda describir la importancia que la espiritualidad tiene en su vida personal. Esto permitiría analizar el impacto puntual de esta variable en la salud de los AM.

En este estudio se conceptualiza la espiritualidad como un conjunto de cogniciones relacionadas con la trascendencia física de la vida y la importancia de éstas en su vida cotidiana.

Estudiar la espiritualidad en los AM en México es importante, ya que puede tener efectos en su salud, pero para demostrar lo anterior se requiere de instrumentos que midan específicamente esta variable. Por lo tanto, los objetivos del presente estudio 
fueron el desarrollo de una instrumento para medir la espiritualidad en AM en México y la evaluación de sus características psicométricas.

\section{MÉTODO}

\section{Participantes}

La muestra estuvo conformada por $355 \mathrm{AM}$ que acudieron a solicitar servicios al Instituto Nacional de las Personas Adultas Mayores (Inapam): 172 AM que solicitaron su credencial en tres centros de atención integral y 182 que acudieron a 11 clubes de la misma institución durante el primer semestre del 2011.

\section{Instrumentos}

Para probar la validez convergente y divergente -asociaciones positivas y negativas respectivamente con otros constructos- (Holton, Bates, Bookter \& Bogdan, 2007) se incluyeron las evaluaciones de depresión y bienestar subjetivo mediante las escalas:

1) Escala de Depresión Geriátrica (EDG) de Yesavage y colaboradores (Yesavage et al., 1983), creada para evaluar los síntomas depresivos en AM. La versión corta consta de 15 reactivos en un formato de respuesta dicotómica. En un estudio con AM en México obtuvo una confiabilidad test-retest de $r=.673$ (GonzálezCelis, 2002); y en otro, también en nuestro país, obtuvo un alfa de Cronbach=.77 (Rivera-Ledesma \& MonteroLópez, 2005). Las opciones de respuesta son presencia/ ausencia de síntomas en la última semana.

2) Escala de Bienestar de Diener y colaboradores (1985), que evalúa la satisfacción con respecto a la vida (el componente cognitivo del bienestar). Cabañero y colaboradores (2004) realizaron algunas modificaciones en la redacción de los reactivos para la población española e identificaron un único factor que explicó el 58.6\% de la varianza, con un alfa de Cronbach $=.82$. La versión tiene cinco opciones de respuesta que van desde "Muy en acuerdo" hasta "Muy en desacuerdo" sin un periodo específico de tiempo. Los reactivos hacen referencia a la satisfacción con respecto a la vida, las condiciones actuales de ésta y la obtención de cosas importantes.

\section{Procedimiento y consideraciones éticas}

La construcción del instrumento se llevó a cabo con base en las aportaciones de AM referentes a la ex- periencia personal de su espiritualidad en la vida cotidiana. Se realizaron tres grupos focales en la sección de la tercera edad del Parque de Chapultepec (Ciudad de México), previa aprobación de las autoridades y posterior invitación a los asistentes regulares de las actividades académicas, deportivas y/o artísticas de la institución para participar voluntariamente. Con una asistencia variable de entre $15 \mathrm{y}$ 25 personas se describió el objetivo de la reunión, se explicó la diferencia entre religiosidad y espiritualidad y se siguió una guía de preguntas relacionadas con la espiritualidad y la experiencia de la misma en la vida cotidiana, para que finalmente los AM expresaran libremente sus experiencias. Las aportaciones de los participantes se analizaron y dividieron en creencias, conductas y emociones, de las cuales sólo se incluyó el apartado de creencias.

Se agruparon las aportaciones de los AM de tal forma que se incluyeron aspectos específicos en reactivos más generales, y de este análisis se conformó un banco de ocho reactivos con un formato de respuesta tipo Likert de cuatro opciones, de "Nunca o casi nunca" a "Todo el tiempo". Para evaluar la comprensión de los reactivos se realizó una prueba piloto en un grupo de 20 AM residentes de una casa asistencial del Instituto Nacional de las Personas Adultas Mayores (Inapam), previa autorización y consentimiento voluntario verbal de los participantes. Posteriormente se realizó la aplicación de la versión final del instrumento de Espiritualidad y de las medidas de Depresión y Bienestar en tres centros de Atención Integral y clubes del Inapam. La aplicación de los instrumentos tuvo dos modalidades: de forma grupal para los grupos ya conformados previamente por la institución, y en entrevista individualizada a quien así lo solicitara al aplicador. En cada administración se informó de forma verbal a los participantes: el objetivo del estudio, la posibilidad de negarse a participar y/o retirarse en cualquier momento, y que la información se manejaría sólo con fines de investigación, de forma anónima.

\section{Análisis estadísticos}

La base de datos se capturó en el programa EPIinfo vers. 6 (Centers for Disease Control and Prevention, 2006) y se analizó con el programa SPSS vers. 13 
(Paquete Estadístico para las Ciencias Sociales, 2000) y AMOS vers. 16 (Arbuckle, 1994,1999). Se empleó el alfa de Cronbach para evaluar la consistencia interna del instrumento. Para evaluar la validez de constructo se realizó el análisis factorial con el método de componentes principales y con rotación varimax, así como un análisis factorial confirmatorio. La validez divergente y convergente se obtuvo por medio de coeficientes de correlación producto-momento de Pearson entre las puntuaciones totales del Índice de Espiritualidad y las medidas de Depresión y Bienestar, respectivamente.

\section{RESULTADOS}

La muestra estuvo conformada por mujeres en un $69.3 \%$ y hombres en un $30.5 \%$, con un promedio de edad de 68 años y una desviación estándar de 7.9 años. El $41 \%$ estaba casada(o) al momento de la aplicación, $31.9 \%$ viuda(o), $10.9 \%$ solteras(os), $8.6 \%$ separadas(os), 4.6 divorciadas(os), y el resto reportó encontrarse en unión libre. La religión predominante fue la católica, con un $85 \%$, seguida por la cristiana, con un $6 \%$, y el $4 \%$ reportó no tener religión. El porcentaje restante se distribuyó entre diferentes doctrinas.

\section{Validez de constructo}

En primer lugar se analizó la discriminación de cada uno de los reactivos a partir de la distribución de las frecuencias en las opciones de respuesta y se encon- tró que los ocho reactivos obtuvieron una distribución de respuestas adecuada.

En el análisis factorial exploratorio todos los reactivos obtuvieron cumunalidades superiores a 0.60 y la prueba de Kaiser-Meyer-Olkin fue de .94, adecuada para continuar con el análisis, el cual arrojó un sólo factor con un valor Eigen $=6.29$ que explicó el 79\% de la varianza.

\section{Análisis factorial confirmatorio}

No fue satisfactorio al incluir los ocho reactivos $\mathrm{X}^{2}$ $(20, \mathrm{~N}=355)=54.99, p<.001)$. En el análisis de correlaciones de los reactivos se encontraron dos con correlaciones superiores a .80 , por lo que se probó un segundo modelo eliminando uno de estos, el cual tampoco fue satisfactorio $\mathrm{X}^{2}(14, \mathrm{~N}=355)=66.43$, $\mathrm{p}<.001)$. Se analizó un tercer modelo con seis reactivos, eliminado ambos $r$ con la asociación de .80, que finalmente resultó satisfactorio $\mathrm{X}^{2}(9, \mathrm{~N}=355)=$ 12.47, $\mathrm{p}=1.881$ ), con una RMSEA $=.033<0.05$, que sugiere que el modelo se ajustó aceptablemente a los datos.

Con los seis reactivos identificados en el análisis confirmatorio se realizó de nuevo el análisis factorial exploratorio; las comunalidades de los reactivos fueron superiores a 0.60 y la prueba de Kaiser-MeyerOlkin fue de .92. El análisis arrojó nuevamente un sólo factor con un valor Eigen de 4.64, que explicó el $77 \%$ de la varianza. En la tabla 1 se presentan los pesos factoriales.

Tabla 1. Análisis factorial de los reactivos del Índice de Espiritualidad

\begin{tabular}{|l|c|}
\hline Reactivos & $\begin{array}{c}\text { Peso } \\
\text { factorial }\end{array}$ \\
\hline 1. Mi espiritualidad me ayuda a definir mis metas. & .95 \\
\hline 2. Mi espiritualidad es importante porque responde mis dudas con respecto al significado de la vida. & .92 \\
\hline 3. Mi espiritualidad me ayuda a entender el significado de las situaciones que vivo. & .90 \\
\hline 4. Me considero una persona espiritual. & .86 \\
\hline 5. Dejar huella en esta vida es una guía para mí. & .83 \\
\hline 6. Creo que hay un "ser superior" que guía mi vida. & .80 \\
\hline
\end{tabular}

La consistencia interna fue alta, con un coeficiente alfa de Cronbach = .94. 
La validez discriminante de grupos contrastados se evaluó con una prueba de promedios de Mann-Whitney para comparar los puntajes globales de la escala, que se situaron por debajo del percentil $25 \mathrm{y}$ arriba del percentil 75 , la cual fue significativa $(z=-14, p<.001)$.

\section{Validez divergente}

Se analizó con la asociación del puntaje de espiritualidad con el puntaje de depresión, y se obtuvo una correlación negativa y estadísticamente significativa $(r=-.20, p=.01)$. Al final, para la validez convergente, se estimó la asociación del puntaje de espiritualidad con el de bienestar subjetivo en su componente cognitivo, resultando positiva y estadísticamente significativa $(r=.11, p=.01)$.

\section{DISCUSIÓN}

La consistencia interna y los análisis de validez del Índice de Espiritualidad demuestran que es un instrumento confiable y válido para evaluar la espiritualidad relacionada con los aspectos cognitivos de la trascendencia de la vida física aplicada a la vida cotidiana en AM. Es un instrumento útil porque es breve, y se distingue de la religiosidad puesto que no hace referencia a doctrina religiosa alguna, además de que se sustituyó el sustantivo "Dios" por "Ser superior", como lo sugieren Bailly y Roussiau (2010) para evitar confusiones entre quienes se consideran espirituales sin ser religiosos. Esto último es importante al reconocer que en México el grupo de AM es predominantemente católico (Rivera-Ledesma \& Montero-López, 2007), y la muestra participante así lo indico (85\%).

A pesar de que la gama de instrumentos disponibles para evaluar la espiritualidad es muy amplia (Sawatzky, 2005), en México el número de instrumentos adaptados a la población es reducido. Además de esta limitación, es muy importante identificar sus características, ya que algunos han sido desarrollados en otros países, y tienen una combinación de aspectos espirituales con religiosos. En este sentido, Daleman y Frey (2004) sugieren que para el campo de la psicología es importante retomar aspectos prácticos de la población para el desarrollo de los instrumentos, más que emplear los ya existentes. En este caso, el presente Índice de Espiritualidad (IE) fue desarrollado desde las aportaciones de los AM en su experiencia cotidiana sobre la espiritualidad, resultando un sólo factor con respecto a la trascendencia de la vida, el cual fue el más relevante para ellos. El IE es una base para ampliar el estudio de la espiritualidad en esta población, de tal forma que aportaciones futuras puedan incorporar reactivos y factores importantes para los AM en México.

La espiritualidad es un constructo que se ha asociado de manera positiva con el bienestar y los indicadores de salud y de forma negativa, con alteraciones emocionales como la depresión en los AM. En este estudio la asociación fue acorde con la dirección y magnitud de otros estudios (Dong \& Eun-Kyoung, 2007; Eun-Kyoung, 2007), y si bien estas asociaciones fueron bajas, están dentro del rango identificado en la literatura: para la depresión el rango de asociación que se ha reportado va de -.10 a -.42 (Daaleman et al., 2004; Dong \& Eun-Kyoung, 2007; Eun-Kyoung, 2007; Underwood \& Teresi, 2002); mientras que para el bienestar va de .10 a .64 (Ardelt, 2003; Bailly \& Roussiau, 2010; Daaleman \& Frey, 2004; Williams \& Sternthal, 2007). Estas variaciones, por supuesto, también dependen en gran medida de las características de la evaluación de espiritualidad. En el presente estudio no se incluyó la medición del componente emocional del bienestar subjetivo, ya que su inclusión será un elemento a futuro en el estudio del bienestar y la espiritualidad.

La evaluación que aporta el Índice de Espiritualidad es útil para incrementar la investigación sobre este constructo en AM mexicanos. Por ello, también se requiere de estudios que incluyan otros o más indicadores de salud para poder identificar la importancia de la misma como un recurso que promueva las capacidades y funcionalidad de los AM, y para desarrollar programas de intervención dirigidos a su fomento y/o mantenimiento (Sawatzky, 2005). Desde esta perspectiva de salud, la espiritualidad puede ser un recurso importante presente en el grupo de AM que permita la comprensión del fenómeno para promover el bienestar y mejorar su salud integral (Powell, 2003). • 


\section{REFERENCIAS}

Aguilar-Navarro, S.G., Fuentes-Cantú, A., Ávila-Funes, J.A. \& García-Mayo, E. J. (2007). Validez y confiabilidad del cuestionario del ENASEM para la depresión en adultos mayores. Salud Pública de México, 49, 356-262.

Arbuckle, J.L. (1994-1999). AMOS [Software]. Chicago: SmallWaters.

Ardelt, M. (2003). Effects of religion and purpose in life on elders' subjective well-being and attitudes toward death. Journal of Religious Gerontology, 14, 55-77.

Bailly, N. \& Roussiau, N. (2010). The Daily Spiritual Experience Scale (DSES): Validation of the short form in an elderly french population. Canadian Journal of Aging, 29, 223-231.

Brady, M. J., Peterman, A.H., Fitchett, G. M.M. \& Cella, D. (1999). A case for including spirituality in quality of life measurement in oncology. Psychooncology, 8, 417-428.

Buendía, J. \& Riquelme, A. (1997). Personalidad, procesos cognitivos y envejecimiento. En J. Buendía (Ed). Gerontología y salud. Perspectivas actuales (pp. 7792). Madrid: Biblioteca Nueva.

Cabañero, M.M.J., Richart, M.M., Cabrero, G.J., Orts, C.M.I., Reig, F.A. \& Tosal, H.B. (2004). Fiabilidad y validez de la Escala de Satisfacción con la Vida de Diener en una muestra de mujeres embarazadas y puérperas. Psicothema, 16, 448-455.

Cella, D.F., Tulsky, D.S., Gray, G., Sarafian, B., Linn E., Bonami, P., et al. (1993). The Functional Assessment of Cancer Therapy Scale: Development and validation of the general measure. Journal of Clinical Oncology, 11, 570-579.

Center for Disease Control and Prevention. (2006). Department of Health and Human Services. Advanced Management and analysis of data using Epi Info for Windows. Center for Disease Control and Prevention.

Cloos, P., Allen, C.G., Alvarado, B.E., Zunzuneguis, M.V., Simeon D.T. \& Eldemire-Shearer, D. (2010) Active ageing: A qualitative study in six Caribbean countries. Aging \& Society, 30, 70-101.

Daaleman, T. P. \& Frey, B.B. (2004). The Spirituality Index of Well-being: A new instrument for health-related quality-of-life research. Annals of Family Medicine, 2, 499-503.
Daaleman, T. P., Frey, B. B., Wallace, D. \& Studenski, S. (2002). The Spirituality Index of Well-being: Development and testing of a new measure. Journal of Family Practice, 51, 11.

Diener, E., Emmons, E.A., Larsen, R.J. \& Griggin, S. (1985). The Satisfaction with Life Scale. Journal of Personality Assessment, 49, 71-75.

Dong, P.Y. \& Eun-Kyoung, O.L. (2007). The impact of religiousness, spirituality, and social support on psychological well-being among older adults in rural areas. Journal of Gerontological Social Work, 281-298.

Ellison, C.W. (1983). Spiritual well-being: Conceptualization and measurement. Journal of Psychology and Theology, 11, 330-340.

Eun-Kyoung, O.L. (2007). Religion and spirituality as predictors of well-being among Chinese American and Korean American older adults. Journal of Religious, Spirituality \& Aging, 19, 77-100.

Fernández-Ballesteros, R. (2009). El bienestar de los mayores. En C. Vázquez \& G. Hervás (Eds). Psicología positiva aplicada (pp. 371-399). Sevilla: Desclée de Brouwer.

Fetzer Institute. (1999). Multidimensional measurement of religiousness/spirituality for use in health research: A report of the Fetzer Institute/National Institute of aging working Group.

González-Celis, R.A.L. (2002). Efectos de intervención de un programa de promoción a la salud sobre la calidad de vida en ancianos. Tesis de Doctorado en Psicología, México, Universidad Nacional Autónoma de México.

González-Celis, R.A.L., Tron, A.R. \& Chávez B.M. (2009). Evaluación de calidad de vida a través del WHOQOL en población de adultos mayores en México. México: Universidad Nacional Autónoma de México, Facultad de Estudios Superiores Iztacala.

Gorge, L.K., Larson, D.B., Koenig, H.G. \& McCullough, M.E. (2000). Spirituality and health: What we know, what we need to know. Journal of Social and Clinical Psychology, 19, 102-116.

Herbert, R.S., Weinstein, E., Martinre, L.M. \& Schulz, R. (2006). Religion, spirituality and the well-being of informal caregivers: A review, critique, and research prospectus. Aging \& Mental Health, 10, 497-520. 
Holton, E. F., Bates, R. A., Bookter, A. I. \& Bogdan, Y. V. (2007). Convergent and divergent validity of the learning transfer system inventory. Human Resource Development Quarterly, 18(3), 385-419. Recuperado el 15 julio de 2012. http://onlinelibrary.wiley.com/ doi/10.1002/hrdq.1210

Idler, E.L., Musick, M.A., Ellison, C.G., George, L.K., Krause, N. \& Ory, M. G. (2003). Measuring multiple dimensions of religion and spirituality for health research: Conceptual background and findings from the 1998 General Social Survey. Research on Aging, 25, 327-365.

Koenig, H.G. (2004). Religion, spirituality, and medicine: Research findings and implications for clinical practice. South Medical Journal. 97, 1194-1200.

Lee, B.Y. \& Newberg, A.B. (2005). Religion and health: A review and critical analysis. Zygon, 40, 443-468.

Lowis, M.J., Eduards, A.C. \& Burton, M. (2009). Coping with Retirement: Well-Being, Health, and Religion. Journal Psychology, 143, 427-448.

Martínez-Mendoza, J.A., Martínez-Ordaz, V.A., EsquivelMolina, C.G. \& Velasco-Rodríguez, V.M. (2005). Prevalencia de depresión y factores de riesgo en el adulto mayor hospitalizado. Revista Médica del Instituto Mexicano del Seguro Social, 45, 21-28.

Mueller, P.S., Plevak, D.J. \& Rummans, T.A. (2001). Religious involvement, spirituality, and medicine: Implications for clinical practice. Clinic Proceedings, 76, 1225-1235.

Organización de las Naciones Unidas. (2002). Informe de la Segunda Asamblea Mundial sobre el Envejecimiento. Organización de las Naciones Unidas.

Oxley, H. (2009). Directorate for Employment, Labour and Social Affairs. OECD Health Working Papers. Organization for Economic Co-operation and Development, 1-32.

Paquete Estadístico para las Ciencias Sociales -SPSS(2000). Para Windows versión 11.5 en español [Software de computadora]. Chicago: SPSS, Inc.

Peterson, C. \& Seligman, M.E.P. (2004). Spirituality. Character strenghts and virtudes. Washington: American Psychological Association Oxford.
Powell, L.H., Shahabi, L. \& Thoresen, C.E. (2003). Religion and spirituality. Linkages to physical health. American Psychologist, 58, 36-52.

Rivera, L.A. (2007). Modelo de intervención racional emotivo para la promoción del ajuste psicológico en el adulto mayor en un contexto religioso. Tesis de doctorado. México: Universidad Nacional Autónoma de México.

Rivera-Ledesma, A. \& Montero-López, M.L. (2005). Espiritualidad y religiosidad en adultos mayores. Salud Mental, 26, 51-58.

Rivera-Ledesma, A. \& Montero-López, M.L. (2007). Medidas de afrontamiento religioso y espiritual en adultos mayores mexicanos. Salud Mental, 30, 39-47.

Sawatzky, R., Ratner, P.A. \& Chiu, L. (2005). A metaanalysis of the relationship between spirituality and quality of life. Social Indicators Research, 72, 153-188.

Smith, T.B., Poll, J. \& McCullough, M.E. (2003). Religiosness and depression: Evidence for a main effect and the moderating influence of stressful life events. Psychological Bulletin, 129, 614-636.

Underwood, L.G. \& Teresi, J.A. (2002). The Daily Spiritual Experience Scale: Development, theoretical description, reliability, exploratory factor analysis, and preliminary construct validity using health-related data. Annals of Behavioral Medicine, 24, 22-33.

Vázquez, C. \& Hervás, G. (2009). Salud positiva: del malestar al síntoma. (2a . Ed.) En C. Vázquez \& G. Hervás. (Eds). Psicología positiva aplicada (pp. 1739). Sevilla: Desclee de Brouwer.

Williams, D.R. \& Sternthal, M. J. (2007). Spirituality, religion and health: Evidence and research directions. Medical Journal of Australia, 186, S47-S50.

Yesavage, J.A., Brink, T.L., Rose, T.L., Lum, O., Huang, V., Adey, M. \& Leirer, V.O. (1983). Development and validation of a Geriatric Depression Screening Scale: A preliminary report. Journal of Psychiatric Research, 17, 37-49.Psicología y Medicina, Madrid. $33,35-41$.
Fecha de recepción: septiembre 2012

Fecha de aceptación: noviembre 2012 